\title{
A Semi-Automated Decision Support Tool for Requirements Trade-off Analysis
}

\author{
Golnaz Elahi \\ Department of Computer Science, University of Toronto \\ gelahi@cs.toronto.edu
}

\author{
Eric $\mathrm{Yu}$ \\ Faculty of Information, University of Toronto, \\ yu@ischool.utoronto.ca
}

\begin{abstract}
System designers and requirements analysts face many competing requirements, such as performance, usability, security, cost, and so forth. To make trade-offs among requirements, ideally analysts would like to quantitatively measure consequences of alternative solutions on requirements. However, during the early stages of requirements and system design, it is hard to quantitatively measure all factors and quantify stakeholders' preferences. The Even Swaps method is a technique developed in management science to assist in multicriteria decision making which allows the use of available but potentially incomplete quantitative and qualitative measures. It teases out the need to elicit importance weights of requirements. Instead, stakeholders are asked how much they would relax one objective to better achieve another. We apply the Even Swaps technique to requirements trade-offs, and supplement it with an algorithm that automates the decision analysis process. The algorithm ns the most distinguishable pair of alternatives and suggests the next requirements to be swapped to stakeholders.
\end{abstract}

Keywords-Requirement Trade-offs, Decision Analysis, Alternative Design Solution, Even Swaps.

\section{INTRODUCTION}

Requirements analysts need to make key decisions early in the project, such as which architectural or design solution to employ [1]. Each alternative solution satis es different functional and non-functional requirements to varying extents. For example, if between two alternatives, one is expensive with a high quality, and the other one has a better price with a low quality, which solution should be picked? Deciding on a solution requires making trade-offs among requirements with respect to consequences of alternatives on the requirements and preferences of stakeholders.

Ideally, analysts would like to quantitatively measure (estimate) consequences of alternative solutions on various requirements. However, while some requirements can be re ned into measurable variables, many non-functional requirements have a soft nature, which makes them hard, if not impossible, to measure. Such requirements are treated as soft goals in some Requirements Engineering (RE) techniques, e.g., $i^{*}[2]$ and Tropos [3]. Goal model evaluation techniques such as [4], [5], [6], enable reasoning about the partial satisfaction of soft goals by propagating qualitative labels such as partially satis ed $(\checkmark \cdot)$, suf ciently satis ed $(\checkmark)$, partially denied $(\Varangle)$, and fully denied $(\chi)$. In some other RE approaches, requirements and alternatives are quanti ed by using ordinal measures or a probabilistic layer for reasoning about partial goal satisfaction [7], [8], [9], [10].
Stakeholders may choose to evaluate some requirements numerically, and some requirements qualitatively, on different scales, e.g., absolute values, percentages, ordinal numbers, or qualitative labels. When different requirements are measured (or estimated) on different scales, normalizing inconsistent types of measures into a single scale is troublesome and may not result in a meaningful utility value. Besides, extracting correct numerical importance weights can be time-consuming and labor-intensive, specially when the number of criteria grows.

In summary, the main problems when making trade-offs among requirements and deciding over alternative design solutions are:

1) Extensive Data Collection: eliciting required information to make an objective decision usually involves an extensive data collection from stakeholders.

2) Manual Prioritization: extracting stakeholders' preferences over multiple criteria in terms of numerical importance weights is error-prone and labor-intensive.

3) Incomparable Scales: aggregating requirements measures in different scales is usually error-prone or not possible.

4) Scalability: the decision problem may become complicated and impossible to be analyzed manually due to numerous requirements and/or alternatives.

Some decision analysis methods evaluate consequences of alternative solutions in terms of precise and meaningful quantitative measures [1], [7], [11]. Some decision analysis approaches such as Analytical Hierarchy Process (AHP) [12] and Even Swaps [13] circumvent the need to measure requirements and consequences of solutions. AHP works based on pairwise comparisons of all decision elements. Even Swaps is a recently introduced decision analysis method in management science that consists of a chain of trading one decision criterion for another. These trades are called swapping. Swaps are even, which means stakeholders are asked to hypothetically improve one criterion, and in return, reduce another one proportionally (evenly).

We adopt the Even Swaps multi-criteria decision analysis approach [13] to make trade-offs among requirements. Since the Even Swaps method works by trading-off one requirement for another, importance weights of requirements are not needed for determining the best solution. Requirements can be evaluated in a mixture of scales and by different measure- 
ment methods. Extensive data collection from stakeholders, as AHP works, are avoided.

This paper introduces a set of guidelines and a tool for tailoring and enhancing the Even Swaps process for requirements trade-off analysis. Although the Even Swaps method solves the problem 1, 2, and 3 (mentioned above), it may fail in practice due to scalability issues: when several software requirements and alternative solutions need to be considered, decision stakeholders can become overwhelmed or confused in making the best swap among numerous possibilities [14]. Selecting two alternatives among many options for the swapping process is also challenging. The main contribution of this paper is introducing an algorithm and tool that:

- Semi-automates the Even Swaps process, in the sense that the process is still interactive with stakeholders while being performed and controlled by an automated algorithm.

- Decides which pair of alternatives to compare in each step.

- Suggests the requirements to swap for analyzing each pair of alternatives.

This paper is organized as follows. In Section II, we introduce an example scenario to illustrate and motivate the problems. Section III introduces basics of the Even Swaps method. Section IV presents details and the algorithm. Section $\mathrm{V}$ presents the results of some case studies of applying the proposed algorithm. In Section VI, some assumptions made in the algorithm are explained and feasibility of the method is discussed. Section VII overviews the related work, and nally Section VIII draws some conclusions and discusses the limitation of the method.

\section{Motivating ExAmple}

The Example Scenario. We motivate our work with a scenario at the Ministry of Transportation, Ontario (MTO), Canada. Traf c Monitoring Centres (TOC) of MTO receive traf $\mathrm{c}$ video feeds from cameras installed in Ontario highways. TOC operators control and manage traf $\mathrm{c}$ by constantly monitoring the ow of the traf c. Consider three hypothetical alternative Intelligent Transportation Systems (ITS): $A_{1}, A_{2}$, and $A_{3}$.

Decision Criteria. To select a solution among alternative ITSs, decision stakeholders need to consider 9 main criteria, indicating costs, security level, usability, and performance of each proposal:

- $T_{1}$ : Time between a change in the traffic and notifying the operator

- $T_{2}$ : Time between requesting a feed and showing the video on the monitor

- $S_{1}$ : Authorized control of cameras

- $P_{1}$ : Percentage of the time cameras are connected

- $G_{1}$ : Easy to view the video feeds
Table I

THE CONSEQUENCE TABLE OF ALTERNATIVE ITS FOR THE MTO SCENARIO

\begin{tabular}{|c|c|c|c|c|c|c|c|c|c|}
\hline & \multicolumn{2}{|c|}{ Minimize } & \multicolumn{5}{|c|}{ Maximize } & \multicolumn{2}{|c|}{ Minimize } \\
\hline Alternative & T1 & $\mathrm{T} 2$ & S1 & P1 & G1 & G2 & G3 & C1 & C2 \\
\hline$A_{1}$ & $2 \mathrm{~ms}$ & $3 \mathrm{~ms}$ & $x$ & $99 \%$ & $\mathrm{H}$ & M & & $\$ 2 \mathrm{~m}$ & $\mathrm{MH}$ \\
\hline A2 & $3 \mathrm{~ms}$ & $4 \mathrm{~ms}$ & $\checkmark$ & $94 \%$ & $\mathbf{L}$ & M & $\sqrt{.}$ & $\$ 1.5 \mathrm{~m}$ & $\mathrm{M}$ \\
\hline A3 & $1 \mathrm{~ms}$ & $2 \mathrm{~ms}$ & $\sqrt{.}$ & $95 \%$ & ML & $\mathbf{L}$ & $\sqrt{.}$ & $\$ 1.8 \mathrm{~m}$ & $M$ \\
\hline
\end{tabular}

- $G_{2}$ : Easy to locate the cameras on the road

- $G_{3}$ : Easy to change the cameras settings

- $C_{1}$ : Cost of implementation

- $C_{2}$ : Maintenance costs

Some of these factors are measurable; for example, the implementation cost of solutions is known and performance can be accurately estimated. On the other hand, the security level and usability of these design solutions are not numerically measurable until the system is actually deployed. $T_{1}$ and $T_{2}$ are measurable performance variables; $S_{1}$ is a security goal that is not directly measurable; $P_{1}$ is security variable; $G_{1}, G_{2}$, and $G_{3}$ are usability goals; and $C_{1}$ and $\mathrm{C}_{2}$ are cost factors.

Consequence Table. We aggregate consequences of alternatives on decision criteria in a table, which we refer to as the consequence table. Table I shows the consequence table for $A_{1}, A_{2}$, and $A_{3}$, and the impacts of these alternatives on the decision criteria. Table I also speci es which criteria need to be minimized or maximized.

Consequence tables may contain heterogeneous data, i.e., different goals can be evaluated on different scales and by different techniques. Some of the criteria can be measurable variables that need to be minimized or maximized. For example, in the case study scenario, stakeholders are able to estimate the Time between a change in the traffic and notifying the operator $\left(T_{1}\right)$ in milliseconds based on the properties and speci cation of $A_{1}$, $A_{2}$, and $A_{3}$. On the other hand, enough information is not available to quantitatively measure the Ease of changing cameras settings $\left(G_{3}\right)$, so they evaluate consequences of $A_{1}, A_{2}$, and $A_{3}$ on $G_{3}$ by using qualitative labels of partially satis ed $\left(\checkmark_{\bullet}\right)$, suf ciently satis ed $(\checkmark)$, partially denied $(\varkappa)$, and fully denied $(x)$. Some requirements are evaluated in the ordinal scale of Low, Medium Low, Medium, Medium High, and High (which are abbreviated as L, ML, M, MH, and $\mathrm{H}$ respectively). We illustrate our method by analyzing trade-offs among ITS requirements and selecting a solution among $A_{1}, A_{2}$, and $A_{3}$.

\section{Basics of the Even Swaps Method}

The Even Swaps decision analysis method extracts stakeholders' value trade-offs, i.e., how much stakeholders would give up on one requirement for better satisfaction of another. For example, suppose two alternative solutions, $A_{1}$ and $A_{2}$ are compared based on their quality and price. The quality 
of $A_{1}$ is High, but it is an expensive solution, costing $\$ 20 \mathrm{~K}$. $A_{2}$ costs $\$ 15 \mathrm{~K}$, but the quality of $A_{2}$ is Medium:

$$
\begin{array}{lll}
A_{1} & \begin{array}{c}
\text { Quality } \\
\text { High }
\end{array} & \text { Price } \\
A_{2} & \text { Medium } & \$ 15 \mathrm{~K}
\end{array}
$$

To select between $A_{1}$ and $A_{2}$, the analyst needs to make a trade-off between quality and price. Making this tradeoff depends on stakeholders' preferences. In this example, analysts need to understand how much stakeholders are willing to pay for a High quality solution. In an even swap, analysts ask stakeholders a hypothetical question: If we could improve the quality of $A_{2}$ from Medium to High, how much you would be willing pay for $A_{2}$ ?

Let us assume stakeholders declare that they would be willing to pay an extra $\$ 3 \mathrm{~K}$ (in total $\$ 18 \mathrm{~K}$ ) for $A_{2}$, if the quality of $A_{2}$ is increased from Medium to High. A solution with High quality and price of $\$ 18 \mathrm{~K}$ is not available. This is a virtual, hypothetical alternative which we call $A_{2}^{\prime}$, and it is as preferred as $A_{2}$, and can be used as its substitute. Now deciding between $A_{1}$ and $A_{2}^{\prime}$ is easier, because they both have a High quality, so the alternatives can be compared with respect to price only. Obviously, $A_{2}^{\prime}$ is a better choice, and since $A_{2}$ is as preferred as $A_{2}^{\prime}$, we can conclude that $A_{2}$ should be preferred to $A_{1}$.

$$
\begin{array}{ccc}
A_{1} & \frac{\text { Quality }}{\text { High }} & \frac{\text { Price }}{\$ 20 \mathrm{~K}} \\
A_{2}^{\prime}=A_{2} & \text { High } & \$ 18 \mathrm{~K}
\end{array}
$$

In an even swap, the decision analyst, collaborating with stakeholders, changes the consequence of an alternative on one requirement, and compensates this change with a preferentially equal change in the satisfaction level of another requirement. Swaps aim to either make criteria irrelevant, in the sense that both alternatives have equal consequences on the criteria, or create a dominant alternative. Alternative $A$ dominates alternative $B$, if $A$ is better than (or equal to) $B$ on every criteria [14]. Irrelevant attributes and dominated alternatives can both be eliminated, and the process continues until only the most preferred alternative remains [14].

Remark In the rest of this paper, when a new concept is de ned or general discussions are given, alternatives are named as $A$ and $B$. The values of $a_{x}$ and $b_{x}$ denote the satisfaction level of the requirements, $g_{x}$, by alternatives $A$ and $B$.

De nition 1. Consider alternative $A$ and two requirements, $g_{x}$ and $g_{y}$, where $A$ satis es $g_{x}$ and $g_{y}$ at the level of $a_{x}$ and $a_{y}$. Swapping two requirements like $g_{x}$ and $g_{y}$ involves changing the value of $a_{x}$ to $a_{x}^{\prime}\left(a_{x} \rightarrow a_{x}^{\prime}\right)$ and compensating this change by modifying $a_{y}$ to $a_{y}^{\prime}\left(a_{y} \rightarrow a_{y}^{\prime}\right)$. We write this swap as:

$$
\left(g_{x}: a_{x} \rightarrow a_{x}^{\prime} \Longleftrightarrow g_{y}: a_{y} \rightarrow a_{y}^{\prime}\right)
$$

The new virtual alternative, $A^{\prime}$, satis es $g_{x}$ and $g_{y}$ at the

\begin{tabular}{|c|c|c|c|c|c|c|c|c|c|}
\hline & \multicolumn{2}{|c|}{ Minimize } & \multicolumn{5}{|c|}{ Maximize } & \multicolumn{2}{|c|}{ Minimize } \\
\hline Alternative & T1 & $\mathrm{T} 2$ & S1 & P1 & G1 & G2 & G3 & C1 & $\mathrm{C} 2$ \\
\hline$A^{\prime} 1$ & $2 \mathrm{~ms}$ & $3 \mathrm{~ms}$ & $x$ & $99 \%$ & $\mathrm{H}$ & $\mathbf{M}$ & $\checkmark$ & $\$ 2 m$ & $\mathrm{MH}$ \\
\hline$A_{2}$ & $3 \mathrm{~ms}$ & $4 \mathrm{~ms}$ & $\checkmark$ & $94 \%$ & $\mathbf{L}$ & $M$ & & $\$ 1.5$ & $M$ \\
\hline
\end{tabular}
level of $a_{x}^{\prime}$ and $a_{y}^{\prime} . A^{\prime}$ is as preferred as the original alternative, $A$.
Table II

SWAPPING $T_{1}$ AND $C_{1}$ FOR $A_{1}$ In THE CASE SCENARIO

Even Swap for Analyzing the Case scenario: Consider consequences of $A_{1}$ and $A_{2}$ in Table I. Stakeholders agree that if the noti cation time $\left(T_{1}\right)$ of alternative $A_{1}$ is increased from 2 milliseconds to 3 , this reduction of performance would be evened out by reducing the cost $\left(C_{1}\right)$ from $\$ 2$ million to $\$ 1.9$ million: $\left(T_{1}: 2 \mathrm{~ms} \rightarrow 3 \mathrm{~ms} \Longleftrightarrow C_{1}: \$ 2\right.$ $\mathrm{m} \rightarrow \$ 1.9 \mathrm{~m}$ ). This swap shows how much stakeholders are willing to relax performance for saving $\$ 0.1$ million. Table II illustrates this swap.

The swap creates a new virtual alternative, $A_{1}^{\prime}$, with revised consequences. The virtual alternative is as preferred as the initial one, and it can be used as a surrogate. The irrelevant goals, i.e., goals on which the consequences of alternatives are indifferent, are removed from the decision process. For example, after swapping $T_{1}$ and $C_{1}, A_{2}$ and $A_{1}^{\prime}$ are indifferent with respect to $T_{1}$ (because the noti cation time of both alternatives is now 3 milliseconds); thus, $T_{1}$ can be removed from the consequence table for the process of deciding between $A_{1}$ and $A_{2}$. By removing $T_{1}$ the problem is simpli ed, in the sense that now instead of 9 criteria, $A_{1}$ and $A_{2}$ are compared with respect to 8 criteria.

The Even Swaps Scalability Problem: When several requirements and alternative solutions need to be considered, determining the right swaps among numerous possibilities is hard for human decision makers [14]. The Even Swaps process does not provide a systematic method or guidelines for stakeholders to carry out the process; thus non-expert users can get stuck in selecting a proper pair of alternatives to analyze or goals to swap. Decision analysts can end up making numerous swaps in a tedious and long process. In Section IV, we propose an algorithm for automating these decisions and suggesting the next step to analysts.

\section{The Automated Even Swaps Tool}

Given a consequence table, the proposed algorithm suggests a chain of swaps to determine the overall best alternative. The algorithm consists of several Even Swaps cycles.

1) In the beginning of each cycle, the algorithm selects a pair of alternatives for the next Even Swaps process (Section IV-A).

2) The automated swap suggestion algorithm takes the pair of alternatives and suggests a chain of swaps to stakeholders intending to nd the preferred alternative in the pair (Section IV-B). 
3) The dominant alternative is kept in the list of solutions, but the dominated alternative will not be considered in the rest of the decision analysis process.

4) The algorithm then selects another pair of alternatives to compare and a new cycle starts. These cycles continue until one alternative remains, which is the best solution overall.

\section{A. Selecting a pair of Alternatives for Even Swaps}

When $n$ alternatives remain in the decision process, there are $\frac{n(n-1)}{2}$ possible pairs of alternatives that can be analyzed in the next Even Swaps process. Does it make a difference which pair is analyzed in the next Even Swaps process? In this section, we discuss two main reasons why the choice of alternatives would affect performance of the algorithm and usability of interactions with stakeholders: 1) Some pairs may require fewer swapping steps to nd the dominant solution than other pairs. 2) Two alternatives that have highly similar consequences on goals will not be easily distinguishable by the stakeholders in the swapping process.

To reduce the number of even swap steps and make the swapping process easier for decision makers, the pair of alternatives for the next Even Swaps process needs to be carefully picked. We set two main criteria for selecting a suitable pair of alternatives: 1) Minimum number of swaps needed to make one alternative dominant, 2) Maximum dissimilarity between consequences of the alternatives (so in the swapping process, stakeholders can easily distinguish the alternatives).

Criterion 1: Minimum number of swaps. The algorithm selects a pair of alternatives that one of them can become dominated with a minimum number of swaps. If $A$ is a better alternatives for $n_{1}$ goals (where $a_{x}>b_{x}$ ) and $B$ is the better alternatives for $n_{2}$ goals (where $b_{x}>a_{x}$ ), then the number of swaps needed to make one of the alternatives dominated is at least $\min \left(n_{1}, n_{2}\right)$.

For example, among three ITS alternatives, $A_{1}, A_{2}, A_{3}$, the possible pairs of alternatives for the Even Swaps process are $\left(A_{1}, A_{2}\right),\left(A_{1}, A_{3}\right)$, and $\left(A_{2}, A_{3}\right)$. Table 2 shows that in the pair of $\left(A_{1}, A_{2}\right), A_{1}$ is a better solution for 5 goals and $A_{2}$ is better for 3 goals. $\left(A_{1}, A_{2}\right)$ and $\left(A_{2}, A_{3}\right)$ both require at least 3 swaps to create a dominated alternative, so we should pick one of these pairs for the next Even Swap cycle. But minimizing the number of swaps is not the only goal; we need to pick a pair of alternatives for the next Even Swaps process that stakeholders are able to easily distinguish their consequences, and swap goals.

Table III

COMPARISON OF ALL POSSIBLE ITS ALTERNATIVE PAIRS.

\begin{tabular}{|l|c|c|c|c|}
\hline Pairs & $A_{1}$ & $A_{2}$ & $A_{3}$ & $\triangle$ \\
\hline$\left(A_{1}, A_{2}\right)$ & 5 & 3 & - & 2.93 \\
\hline$\left(A_{1}, A_{3}\right)$ & 5 & - & 4 & 2.99 \\
\hline$\left(A_{2}, A_{3}\right)$ & - & 3 & 4 & 2.57 \\
\hline
\end{tabular}

Criterion 2: Maximum divergence between consequences. The underlying idea of this criterion is to select a pair of alternatives that their consequences are easily differentiable. Thus, when stakeholders are asked to make a swap, consequences of alternatives are divergent enough that stakeholders can make an explicit trade-off between the requirements. To illustrate this idea, consider the ITS example where we need to determine the dominant solution in the pair of $A_{2}$ and $A_{3}$ by swapping a usability goal, $G_{2}$ and an availability percentage, $P_{1}$. If stakeholders are asked how much they would sacri ce on $G_{2}$, for increasing $P_{1} 1 \%$ from $94 \%$ to $95 \%$, they may not nd this $1 \%$ improvement enough to relax the satisfaction level of $G_{2}$. This problem stems from the insigni cant difference between $A_{2}$ and $A_{3}$ 's consequence on $P_{1}: 94 \%$ is barely distinguishable from $95 \%$.

In order to measure how distinguishable a pair of alternatives are we calculate a "distance factor", $\triangle(A, B)$ between consequences of $A$ and $B$ on goals:

$$
\triangle(A, B)=\sum_{x=1}^{m} \frac{\left|a_{x}-b_{x}\right|}{\max _{g_{x}}}
$$

The delta of consequences on goal $g_{x}\left(\left|a_{x}-b_{x}\right|\right)$ is divided by the maximum satisfaction level on $g_{x}$ in the consequence table $\left(\max _{g_{x}}\right)$. In this way, the delta values are normalized to a value in the range of 0 to 1 , so they can be summed up into one value. For example, the distance factor of $\left(A_{1}, A_{2}\right)$ is calculated as:

$$
\begin{aligned}
& \triangle\left(A_{1}, A_{2}\right)=\frac{|2-3|}{3}+\frac{|3-4|}{4}+\frac{|\boldsymbol{X}-\sqrt{ }|}{\checkmark}+\frac{|99-94|}{99}+\frac{|H-L|}{H}+ \\
& \frac{|M-M|}{M}+\frac{|\mathcal{V}-\sqrt{ } \cdot|}{\checkmark}+\frac{|2-1.5|}{2}+\frac{|M H-M|}{M H}
\end{aligned}
$$

Calculation Method: Calculating deltas for absolute measures is straightforward. However, measuring the differences of two qualitative labels as $|x-\checkmark|$ is troublesome, because these labels do not bear a quantitative implication. Based on the relative rankings $(\checkmark>\checkmark \cdot>x>x)$ we can conclude $|\checkmark-x|>|V-x|$. Semantically, $\checkmark$ is the opposite of $x$ and $\checkmark$. is the opposite of $x$. Thus, we assume $|\checkmark-\underline{x}|=|\sqrt{ }-x|$ and $|\checkmark-\mathfrak{v}|=|\not{x}-x|$. In order to calculate the distance factor, we assign an arbitrary numerical representation to $\checkmark, V_{\text {. }}, \boldsymbol{x}$, and $\boldsymbol{x}$ that solves the ordering relations we assumed. For example, $+2,+1,-1,-2$ or $4,3,2,1$ are possible arbitrary representations for the qualitative labels of $\checkmark, \sqrt{ }$.,.$\times$, and $\times$ respectively (we use 4 , $3,2,1$ in the algorithm).

In the scale of Low to High, Medium is in the middle of Low and High (their average). Medium Low and Medium High are another ner grained middle values. Thus, the values of Low to High can be represented by any interval scale that ts the discussed relations (we used the interval scale of 1 to 5 in the calculations).

Imprecision: The underlying purpose of calculating the deltas is to measure the extent of dissimilarity of two alternatives on each goal. Note that normalizing these values 
does not provide us with means for calculating a meaningful utility for the alternatives. For example, the percentage of the time that cameras are connected $\left(P_{1}\right)$ is $95 \%$ for $A_{3}$ (Table I); however, the utility of $A_{3}$ on $P_{1}$ is not re ected by the relation of $95 \%$ to the practical maximum connectivity percentage $(99 \%)$ or absolute maximum connectivity (100\%). If availability of the connection to cameras is highly critical, the utility of $A_{2}$ on $P_{1}$ is not about $\frac{95}{99}$, and the utility of any connectivity lower than $99 \%$ (for example) could even be zero.

With the same argument, one can claim that Formula 1 does not measure the dissimilarity of two alternatives correctly, because if the utility of $95 \%$ is zero, the utility of $A_{2}$ on $P_{1}(94 \%)$ is also zero. Although on the face are dissimilar, $A_{2}$ and $A_{3}$ are indifferent with respect to $P_{1}$. Nevertheless, the distance factors of the pairs of alternatives are comparable because all distance factors are calculated with equal imprecision.

For example, $\triangle\left(A_{1}, A_{2}\right)=2.93$ and $\triangle\left(A_{2}, A_{3}\right)=2.57$ (Table 2), so we conclude that consequences of $A_{1}$ and $A_{2}$ are more dissimilar than the consequences of $A_{2}$ and $A_{3}$, although both numbers are inaccurate. In the next section, we introduce the automated swap suggestion algorithm to select the dominant solution in the pair of $\left(A_{1}, A_{2}\right)$.

\section{B. Automatically Suggesting Swaps}

In each cycle of the algorithm, two alternatives are analyzed by applying a chain of swaps, until one becomes dominated. One of the main goals of the proposed algorithm is to reduce the number of swapping steps through reusing swaps from previous cycles. We develop 4 rules for automatically generating reusable swaps that are also easy to make. In this section, these rules are explained and justi ed.

1) Creating a Dominant Alternative: Swaps make one of the decision criteria irrelevant, which helps remove one goal from the decision problem in each step. Removing criteria one by one is a time-consuming approach to apply the Even Swaps process. For example, consider an alternative such as $A$ that is dominant for $n$ goals like $g_{1}, g_{2}, \ldots g_{n}$, and alternative $B$ that is a better solution only with respect to one goal, like $g$. We need to remove $g$ by swapping it with one of those $n$ goals. By removing $g$, solution $A$ might still be dominant with respect to all goals $\left(g_{1}, g_{2}, \ldots g_{n}\right)$. However, swapping any two goals from $g_{1}, g_{2}, \ldots g_{n}$ will not change the situation between $A$ and $B$ and neither of them becomes dominated with respect to all relevant and remaining goals. By following rule 1, the algorithm suggests a swap that aims making one of the alternatives dominant:

Rule 1, create a dominant alternative: Given a set of goals $G=\left\{g_{1}, g_{2}, \ldots g_{m}\right\}$, if consequences of $A=$ $\left\{a_{1}, a_{2}, \ldots a_{m}\right\}$ and consequences of $B=\left\{b_{1}, b_{2}, \ldots b_{m}\right\}$, and $n_{1}=$ number of goals where $a_{x}>b_{x}$ and $n_{2}=$ number of goals where $b_{x}>a_{x}$ (for $1 \leq x \leq m$ ), then $n_{1} \times n_{2}$ swaps exist that could potentially reduce the number of steps of the Even Swaps process for making an alternative dominant.

For example, between the pair of $A_{1}$ and $A_{2}$, with respect to $T_{1}, T_{2}, P_{1}, G_{1}$, and $G_{3}, A_{1}$ is a better solution, and with respect to $S_{1}, C_{1}$, and $C_{2}, A_{2}$ is the better solution. Note that the satisfaction level of both alternatives on $G_{2}$ is Medium, so $G_{2}$ is an irrelevant goal for deciding between $A_{1}$ and $A_{2}$.

To minimize the number of swaps for $A_{1}$ and $A_{2}$, a goal such as $g_{x}$ needs to be swapped with a goal such as $g_{y}$, where $g_{x} \in\left\{S_{1}, C_{1}, C_{2}\right\}$ and $g_{y} \in\left\{T_{1}, T_{2}, P_{1}, G_{1}, G_{3}\right\}$. Therefore, 15 tuples in the form of $\left(g_{x}, g_{y}\right)$ exists as the candidate pair of goals to be swapped. By making a swap between such goals, one of three goals among $S_{1}, C_{1}$, and $C_{2}$ becomes irrelevant; thus, in the next step, at least 2 more swaps are needed to make one of the alternatives dominant.

2) Suggesting the Most Reusable Swap: When stakeholders make a swap, their value trade-offs can be reused for another alternative, without further consultation with human stakeholders (if the goals are the same and the satisfaction level of goals in the swap are the same with the new alternative).

Rule 2, pick the most reusable swaps: When there are several goal tuples like $\left(g_{x}, g_{y}\right)$ for the next swap, by applying rule 2 , the algorithm selects the most reusable swap for the next step. The notion of reusable swap is formally de ned as follows:

De nition 2. Consider an alternative, $A$, and goals $g_{x}$ and $g_{y}$, where $A$ satis es $g_{x}$ and $g_{y}$ at the level of $a_{x}$ and $a_{y}$. The swap $\left(g_{x}: i_{x} \rightarrow i_{x}^{\prime} \Leftrightarrow g_{y}: i_{y} \rightarrow i_{y}^{\prime}\right)$ is reusable for alternative $A$ on goals $g_{x}$ and $g_{y}$, if $i_{x}=a_{x}$, and $i_{y}=a_{y}$.

None of those 15 candidate swaps for $A_{1}$ and $A_{2}$ are reusable for the consequences of $A_{3}$. To illustrate the concept of reusability, consider three hypothetical solutions $A$, $B$, and $C$, and three goals $g_{1}, g_{2}$, and $g_{3}$, which we aim to maximize. Let us assume consequences of alternatives on the goals are as:

$$
A=\{H, M, L\}, \quad B=\{M H, L, H\}, \quad C=\{H, M, M L\}
$$

Consider the swap $\left(g_{1}: H \rightarrow M H \Leftrightarrow g_{2}: M \rightarrow x\right)$ for deciding between $A$ and $B$. Assume the stakeholder has previously agreed to reduce the satisfaction level of $A$ on $g_{1}$ from $\mathrm{H}$ to $\mathrm{MH}$, and in return, increase the satisfaction level of $g_{2}$ from $\mathrm{M}$ to $x=H$. This swap is reusable for deciding between $B$ and $C$ as well, where the satisfaction level of $C$ on $g_{1}$ and $g_{2}$ can be modi ed according to this swap without the need for asking another swap from stakeholders.

3) Suggesting Easy Swaps: In addition to considering swaps reusability, the algorithm suggests swaps that decision stakeholders would be willing to make. Hammond et al. [13] suggest making the easiest swaps rst, e.g., money is an easy goal to swap. What would make a swap easy for stakeholders? For example, stakeholders may easily agree to increase improve a goal that is not suf ciently satis ed 


\begin{tabular}{|c|c|c|c|c|c|c|c|c|c|}
\hline $\begin{array}{l}\text { Swaps in the } \\
\text { form of (gx, gy): }\end{array}$ & & Sy & $g_{x}$ & gy & & & gy & & gx \\
\hline Goals & T1 & T2 & S1 & P1 & G1 & G2 & G3 & C1 & C2 \\
\hline $\begin{array}{c}\text { Distance factors } \\
\text { of } A 1 \text { and } A 2 \\
\text { on goals }\end{array}$ & $\begin{array}{c}\frac{|.5-.33|+.5}{1} \\
=0.67\end{array}$ & $\begin{array}{c}\frac{|.33-.25|+.33}{.5} \\
=0.82\end{array}$ & $\begin{array}{c}\frac{|1-4|+1}{4} \\
=1\end{array}$ & $\begin{array}{c}\frac{|99-94|+99}{99} \\
=1.05\end{array}$ & $\begin{array}{l}\frac{|5-1|+5}{5} \\
=1.8\end{array}$ & $\begin{array}{c}\frac{|3-3|+3}{3} \\
=1\end{array}$ & $\begin{array}{l}\frac{|4-3|+4}{4} \\
=1.25\end{array}$ & $\begin{array}{c}\frac{|.5-.66|+.5}{=.66} \\
=1\end{array}$ & $\begin{array}{c}\frac{|.25-.33|+.25}{.33} \\
=1\end{array}$ \\
\hline
\end{tabular}

Figure 1. Distance factors of alternatives $A_{1}$ and $A_{2}$ on ITS goals

and compensate it with decreasing the satisfaction level of a requirement that is highly satis ed, intending to reach a balance among software requirements.

In addition, if consequences of two alternatives on the rst goal of the swap are close, with an insigni cant change on the consequence of one alternative on this goal, it can become an irrelevant goal. In this way, the goals that do not differentiate alternatives are eliminated from the problem earlier. Since these goals may not affect the nal decision dramatically, they can be eliminated earlier in the decision analysis process.

As the compensation of the insigni cant change to the rst goal, the algorithm searches for a goal to swap, on which consequences of alternatives are highly differentiable. To understand the philosophy of this rule, assume $A$ is the dominant alternative (in the pair of $A$ and $B$ ) with respect to all goals, except a goal such as $g_{x}$. By removing $g_{x}$ in a swap, $A$ can become the overall dominant. Suppose the satisfaction level of $g_{x}$ is increased, and as an even compensation, the satisfaction level of another goal such as $g_{y}$ is reduced: $\left(g_{x}: a_{x} \rightarrow a_{x}^{\prime} \Leftrightarrow g_{y}: a_{y} \rightarrow a_{y}^{\prime}\right)$, where $a_{x}^{\prime}>a_{x}$ and $a_{y}^{\prime}<a_{y}$. Now, $g_{x}$ has become irrelevant and is removed from the list of decision criteria, but it is possible that $A_{i}^{\prime}$ is now dominant with respect to all goals except $g_{y}$. This means the value of $a_{y}$ has reduced so much that now $B$ is the dominant one with respect to $g_{y}$. In this situation, the swap has not been effective for creating a dominance situation and still another swap is needed to make solution $A$ dominant. If consequences of $A$ on the $g_{y}$ is much higher than $B$, it is more probable that after reducing $a_{y}$ to $a_{y}^{\prime}$, still $A^{\prime}$ is the better solution with respect to $g_{y}$.

Rule 3, make the easiest swap: This rule summarizes the characteristics which we discussed above. When comparing the pair of alternatives $A$ and $B$, two goals such as $g_{1}$ and $g_{2}$ should be swapped where the satisfaction level of $A$ on $g_{1}$ is minimum compared to any other goal, and at the same time, $\left|a_{1}-b_{1}\right|$ is minimum. The second goal, $g_{2}$, has the highest satisfaction level compared to other goals, and at the same time $\left|a_{2}-b_{2}\right|$ is maximum.

In this way, since the satisfaction level of $g_{1}$ is relatively low, and the satisfaction level of $g_{2}$ is relatively higher than other goals, stakeholders probably agree to increase consequences of $A$ on $g_{1}$ (from $a_{x}$ to $b_{x}$ ) and compensate it with reducing the satisfaction level of $g_{2}$. In addition, since consequences of $A$ and $B$ on $g_{1}$ are close, with an insigni cant change on $g_{1}$, it can become an irrelevant goal.
Consequences of $A$ and $B$ on $g_{2}$ are divergent $\left(\left|a_{2}-b_{2}\right|\right.$ is maximum); thus, by applying the swap and reducing the satisfaction level of $g_{2}, A$ (or $B$ ) remains dominant.

How these objectives can be formulated? We de ne a distance factor for alternatives for a given goal, which aggregates these desired properties into a value. The distance factor on the goal $g_{x}$ is $\triangle\left(A, B, g_{x}\right)$ and calculated as:

$$
\triangle\left(A, B, g_{x}\right)=\frac{\left|a_{x}-b_{x}\right|+a_{x}}{\max _{g_{x}}}
$$

where $\max _{g_{x}}$ is the maximum satisfaction level of $g_{x}$ in the consequence table.

The purpose of rule 3 is to swap a goal with minimum satisfaction level (minimum $a_{x}$ in formula (2)) and the smallest distance from $B$ (minimum $\left|a_{x}-b_{x}\right|$ in formula (2)) with a goal that has maximum satisfaction level and maximum distance from $B$.

This formula, therefore, does not re ect the minimum and maximum satisfaction for the goals and variables that stakeholders aim to minimize. For such goals, the higher $a_{x}$ is, the less the satisfaction level of $g_{x}$ is. Hence, if $g_{x}$ is a goal or variable that needs to be minimized, then the algorithm replaces $a_{x}$ with $\frac{1}{a_{x}}$ in formula (2). For brevity, we write $\operatorname{Sat}(g, A)$ to denote the satisfaction level of goal $g$ by alternative $A$. For example, $\operatorname{Sat}\left(T_{1}, A_{1}\right)=\frac{1}{2}$, $\operatorname{Sat}\left(T_{1}, A_{2}\right)=\frac{1}{3}$, and $\operatorname{Sat}\left(T_{1}, A_{3}\right)=\frac{1}{1}$, so $\max _{T_{1}}=1$. To be able to apply this formula, we assume that all goals and variables have a none-zero satisfaction level.

Figure 1 shows the distance factors of $A_{1}$ and $A_{2}$ on the goals in Table I. For example, the distance factors of $A_{1}$ and $A_{2}$ on $T_{1}$ and $S_{1}$ are:

$$
\begin{aligned}
& \triangle\left(A_{1}, A_{2}, T_{1}\right)=\frac{\left|\frac{1}{2}-\frac{1}{3}\right|+\frac{1}{2}}{1}=0.67 \\
& \triangle\left(A_{1}, A_{2}, S_{1}\right)=\frac{|1-4|+1}{4}=1
\end{aligned}
$$

Previously, we identi ed 15 candidate swaps for analyzing $A_{1}$ and $A_{2}$ : these 15 candidates are tuples like $\left(g_{x}, g_{y}\right)$, where $g_{x}$ can be any of $S_{1}, C_{1}, C_{2}$, and $g_{y}$ can be any of $T_{1}$, $T_{2}, P_{1}, G_{1}, G_{3}$. Based on rule $3, g_{x}$ shall have the minimum distance factor. Since the distance factor of $A_{1}$ and $A_{2}$ on $S_{1}$, $C_{1}$, and $C_{2}$ is $1, g_{x}$ can be any of these three goals. Rule 3 also indicates that $g_{y}$ shall have the maximum distance factor; therefore, $G_{1}$ is the best choice for $g_{y}$ (Figure 1. In sum, by applying rule 3 , candidate goal tuples for the next swaps are reduced to 3 tuples: $\left(C_{1}, G_{1}\right),\left(C_{2}, G_{1}\right),\left(S_{1}, G_{1}\right)$.

Which of these three possible goals should be suggested 
for the next swap? The nal rule for suggesting the next swap is selecting goals that are measured in more granular and accurate scales, because dealing with tangible factors is easier for stakeholders. For example, costs in terms of money is more tangible than the usability level expressed as Medium, Low, High. In this regard, rule 4 states:

Rule 4, pick preferred scales: Goals that are measured in absolute values are preferred to the goals measured in percentages, percentages are preferred to ordinal values, and the least preferred scale is qualitative labels.

For example, based on rule 4 , the scale of $C_{2}$ is preferred to the scale of $S_{1}$ and the scale of $C_{1}$ is preferred to $C_{2}$, since $C_{1}$ is an absolute value (million dollars). Therefore, among three options $\left(\left(C_{1}, G_{1}\right),\left(C_{2}, G_{1}\right),\left(S_{1}, G_{1}\right)\right)$, the algorithm selects $C_{1}$ and $G_{1}$ to be swapped, and asks the value of $x$ in the swap: $\left(C_{1}: \$ 2 m \rightarrow \$ 1.5 \mathrm{~m} \Leftrightarrow G_{1}: H \rightarrow x\right)$.

The aim of this swap is to elicit how much stakeholders would give up on $G_{1}$ for paying a lower price $\left(C_{1}\right)$, so $C_{1}$ is reduced to from $\$ 2 \mathrm{~m}$ to $\$ 1.5 \mathrm{~m}$, and as a compensation, $G_{1}$ is decreased from $H$ to a lower level $(x)$. Suppose the stakeholder agrees with $x=L$. The consequence table is revised with these new values (the satisfaction level of $G_{1}$ under the consequence of $A_{1}$ is now $\mathrm{L}$, and the cost of alternative $A_{1}$ is now $\$ 1.5$ million). $C_{1}$ can be removed from the list of goals that need to be considered for deciding between $A_{1}$ and $A_{2}$. (Not deliberately, the swap makes $G_{1}$ irrelevant as well.)

4) Reusing Dominance Situations: Once an alternative is decided as the dominant one in a pair, this knowledge can be reusable for deciding between other pairs of alternatives without the need to the even swaps process. For example, consider four hypothetical solutions $A, B, C$, and $D$ and three goals $g_{1}, g_{2}$, and $g_{3}$, where consequences of alternatives on the goals are as:

$$
\begin{aligned}
& A=\{H, M, L\}, \quad B=\{M H, L, H\} \\
& C=\{H, M, M L\}, \quad D=\{M, L, H\}
\end{aligned}
$$

Assume by applying a chain of swaps, the algorithm concludes that $A>B$, then we can conclude that $C>D$ as well, because $C$ dominates $A$ on every goal, and $B$ dominates $D$ on every goal. Since dominance is a transitive relation, we can conclude that $C$ dominates $D$ :

Reuse the dominance situation: In general, for four alternatives $A, B, C$, and $D$, where the consequences of alternatives on goals $G=\left\{g_{1}, g_{2}, \ldots, g_{m}\right\}, A=$ $\left\{a_{1}, a_{2}, \ldots, a_{m}\right\}, B=\left\{b_{1}, b_{2}, \ldots b_{m}\right\}, C=\left\{c_{1}, c_{2}, . . c_{m}\right\}$, and $D=\left\{d_{1}, d_{2}, \ldots d_{m}\right\}$. If $A>B$, then $C>D$ too, iff:

$$
\begin{aligned}
& -\forall\left(a_{x}, c_{x}\right) \quad c_{x} \geq a_{x} \\
& \text { - } \forall\left(b_{x}, d_{x}\right) \quad d_{x} \leq b_{x} \\
& (1 \leq x \leq m)
\end{aligned}
$$

\section{The Automated Swaps Suggestion Algorithm}

Assume a consequence table that includes a set of goals $G=\left\{g_{1}, g_{2}, \ldots g_{m}\right\}$, and two alternatives $A$ and $B$ to be analyzed in the next cycle of the algorithm. The algorithm suggests swaps, gets the stakeholders input, applied the swap, and stores the swaps in a Knowledge Base (KB). In each step, the algorithm keeps the tuples of candidate goals for the next swap in temporary array lists of $L, L^{\prime}, L^{\prime \prime}, L_{f}$. The process for determining the optimum solution between $A$ and $B$ consists of 8 main steps as follows.

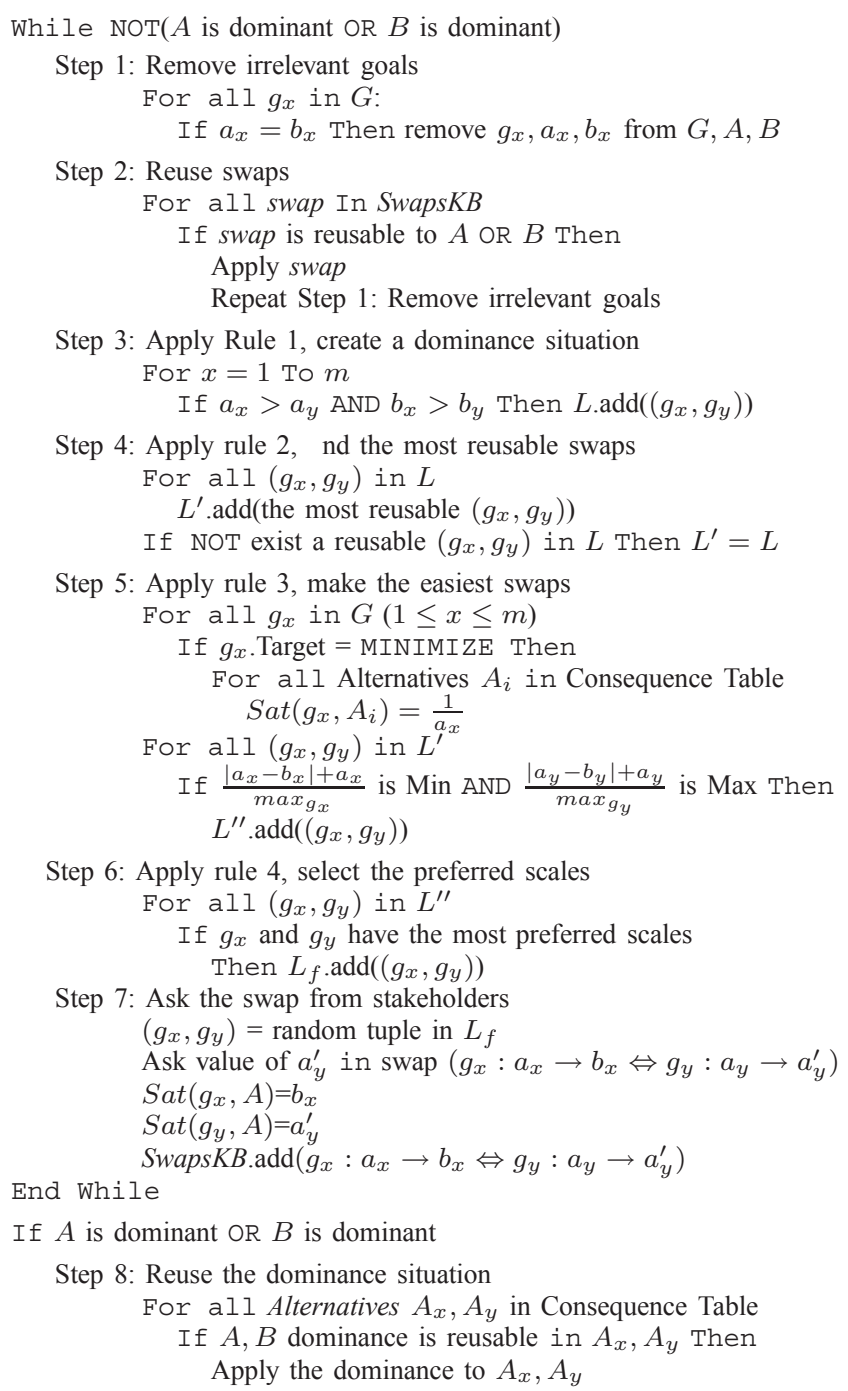

\section{CAse Studies And Comparison}

We illustrate the use of the proposed method by analyzing a number of case studies including the alternative ITS systems. We focus on evaluating three characteristics of the method: 1) number of swap enquiries asked from stakeholders to determine the dominant alternative 2) the initial data needed to make the decision 3) validity of the decision made by the algorithm.

Case study 1: alternative ITS systems at MTO. In the previous section, we explained how the algorithm selects the pair of $A_{1}$ and $A_{2}$ for the rst Even Swaps process. First, the algorithm suggests and applies $\left(C_{1}: \$ 2 m \rightarrow\right.$ $\left.\$ 1.5 m \Leftrightarrow G_{1}: H \rightarrow L\right) . C_{1}$ and $G_{1}$ become irrelevant, 
Table IV

SUMMARY OF CASE STUDIES

\begin{tabular}{|c|c|c|c|c|c|c|}
\hline Case Study & \# alts & \# goals & Initial Data & $\begin{array}{l}\text { Number of } \mathrm{W} \\
\text { queries }\end{array}$ & $\begin{array}{l}\text { Correctness of } \\
\text { algorithm's decision }\end{array}$ & $\begin{array}{l}\text { Number of } \\
\text { swap queries }\end{array}$ \\
\hline $\begin{array}{l}\text { ITS systems } \\
\text { at MTO }\end{array}$ & 3 & 9 & $\begin{array}{c}\text { W: not needed } \\
\text { S: Qual. or Quant data }\end{array}$ & Not needed & Not known & 10 \\
\hline $\begin{array}{l}\text { Scenario } \\
\text { in [11] }\end{array}$ & 4 & 5 & $\begin{array}{l}\text { W: Quant data needed } \\
\text { S: Quant data needed }\end{array}$ & $\begin{array}{c}\text { AHP: } 10 \\
\text { Direct assessment: } 5\end{array}$ & Correct decision made & 2 \\
\hline $\begin{array}{l}\text { Scenario } \\
\text { in [7] }\end{array}$ & 3 & 5 & $\begin{array}{l}\text { W: Quant data needed } \\
\text { S: Quant data needed }\end{array}$ & $\begin{array}{c}\text { AHP: } 10 \\
\text { Direct assessment: } 5\end{array}$ & Correct decision made & 3 \\
\hline \multicolumn{5}{|c|}{ Decision analysis effort in the original scenarios } & \multirow{2}{*}{\multicolumn{2}{|c|}{$\begin{array}{c}\text { Results of applying the automated } \\
\text { Even Saps method }\end{array}$}} \\
\hline \multicolumn{3}{|c|}{ W: Importance weight } & \multicolumn{2}{|c|}{ Qual. : Qualitative } & & \\
\hline S: Satisfactic & level & f goals & \multicolumn{2}{|l|}{ Quant. : Quantitative } & & \\
\hline
\end{tabular}

and the algorithm removes both. The process continues with suggesting the next swaps:

Relevant goals $=\left\{T_{1}, T_{2}, S_{1}, P_{1}, G_{3}, C_{2}\right\}$

Consequences of $A_{1}^{\prime}=\{2 m s, 3 m s, \times, 99 \%, \sqrt{ }, M H\}$

Consequences of $A_{2}=\{3 m s, 4 m s, \sqrt{ }, 94 \%, \sqrt{ }$.,$M\}$

Next suggested swap: $\left(C_{2}: M H \rightarrow M \Leftrightarrow G_{3}: \checkmark \rightarrow x\right)$

Stakeholders respond $x=\sqrt{ }$., so $C_{2}$ and $G_{3}$ can be removed Relevant goals $=\left\{T_{1}, T_{2}, S_{1}, P_{1}\right\}$

Consequences of $A_{1}^{\prime}=\{2 m s, 3 m s$, X, $99 \%$, $\}$

Next suggested swap: $\left(S_{1}: \mathcal{X} \rightarrow \boldsymbol{V} \Leftrightarrow P_{1}: 99 \% \rightarrow x\right)$

Stakeholders respond $x=90 \%$, so $S_{1}$ can be removed Relevant goals $=\left\{T_{1}, T_{2}, P_{1}\right\}$

Consequences of $A_{1}^{\prime}=\{2 m s, 3 m s, 90 \%\}$

Next suggested swap: $\left(P_{1}: 90 \% \rightarrow 94 \% \Leftrightarrow T_{2}: 3 m s \rightarrow x\right)$

Stakeholders respond $x=4 \mathrm{~ms}$, so $P_{1}$ and $T_{2}$ can be removed Relevant goals $=\left\{T_{1}\right\}$

Consequences of $A_{1}^{\prime}=\{2 m s\}$ and Consequences of $A_{2}=\{3 m s\}$

$A_{1}$ is the dominant alternative

The consequence table is reset to the initial values

In the pair of $A_{1}$ and $A_{2}$, the dominant solution is $A_{1}$. Then, the pair of $A_{1}$ and $A_{3}$ are analyzed by the automated Even Swaps process. By suggesting and applying 6 swaps, $A_{3}$ is determined as the dominant one. Although $A_{2}$ is the most economical solution, it was eliminated because of its poor usability $\left(G_{1}\right)$ and unreliable connectivity to the cameras $\left(P_{1}\right) . A_{3}$ is more economical than $A_{1}$ and has the best performance $\left(T_{1}\right.$ and $\left.T_{2}\right)$, and although the usability of $A_{3}$ is mediocre, overall, $A_{3}$ is preferred to $A_{1}$.

In total, the algorithm asked 10 swap queries. In this way, non-numerical data is systematically handled, while quantitative methods such as Utility Theory [15] are not able to accurately aggregate non-numerical input into the utility value. In addition, importance weights of the criteria were not needed, while preferences are directly considered in the nal decision.

Case study 2: analyzing the decision scenarios in [11], [7]. We repeat the analysis of example scenarios in two requirements decision analysis proposals [11], [7] by applying the automated Even Swaps method. We compare the results of the methods in the original contributions with the results of the proposed algorithm. This comparison indicates whether the algorithm determines the best solution correctly and with reasonable number of swap enquiries.

In [11], four alternatives are compared with respect to 5 main criteria: Functionality, Maintenance, Project,
Process, Schedule and Cost. The assessments of alternatives based on these criteria are provided quantitatively in the original contribution. By using different preference modeling methods, such as AHP [12], importance weights of criteria are also extracted.

Given the numerical consequences of alternatives on the criteria, we applied the automated Even Swaps algorithm to determine the overall best solution in the scenario originally presented in [11]. In order to swap goals, we use the numerical importance weights in the original example to interpret which goals are preferred to others. For example, the importance weights of Time and Functionality in [11] are 0.25 and 0.17 , so Time is more important than Functionality. Thus, in the swap (Time : $0.2 \rightarrow 0.4 \Leftrightarrow$ Functionality : $0.9 \rightarrow x$ ), increasing Time from 0.2 to 0.4 shall be compensated with reducing Functionality from 0.9 to a level $x$, where $0.9-x>0.4-0.2$. With a small change to Time from 0.2 to 0.4 , Functionality needs to be reduced relatively more than 0.2 , thus $x$ can be $=0.6$.

Table 2 summarizes and compares the results of applying the automated Even Swaps algorithm with the results in the original contributions [11]. The proposed algorithm, in total, asks the stakeholders 2 swap queries, while in order to extract the numerical importance weights of 5 criteria by direct assessment at least 5 queries are needed, and by using AHP, at least 10 AHP comparison must be made by stakeholders.

We also repeated the decision analysis example in [7] using the automated Even Swaps algorithm. In [7], 3 alternatives are compared with respect to 5 goals. By applying 3 swaps, the algorithm picks the best solution correctly. In both of these studies, the automated swap suggestion algorithm reduces the burden and complication of extracting importance weights and determines the best alternative correctly (compared to the nal conclusions in the original work in [11], [7]).

\section{DISCUSSION}

The Notion of Swap Reusability. Marginal Rate Substitution (MRS) indicates the maximal amount of a goal satisfaction level that stakeholders are willing to sacri ce 
for a unit of increase in another goal. Even swaps implicitly capture the MRSs for the goals that stakeholders swap. Generally, MRSs of two goals at two different satisfaction levels can be different [16]. The notion of swaps reusability which we discussed in the previous section is based on assuming that MRSs are static, and the consequence changes made in an even swap depend on the satisfaction levels of the goals; this restricts the reusability of swaps. In what follows, we explain the underlying reasons for assuming that MRSs are static.

In the swap $\left(g_{x}: i_{x} \rightarrow i_{x}^{\prime} \Leftrightarrow g_{y}: i_{y} \rightarrow i_{y}^{\prime}\right)$, changing $g_{x}$ by $d_{x}$ levels $\left(d_{x}=\left|i_{x}^{\prime}-i_{x}\right|\right)$ is compensated with changing $g_{y}$ by $d_{y}$ levels $\left(d_{y}=\left|i_{y}-i_{y}^{\prime}\right|\right)$. Here, stakeholders have a mental utility function like $\mathrm{F}$, which helps them balance $\left|F\left(i_{x}^{\prime}\right)-F\left(i_{x}\right)\right|$ with $\left|F\left(i_{y}\right)-F\left(i_{y}^{\prime}\right)\right|$. However, the utility function, $F$, is not linear; hence, in a situation where the satisfaction level of $g_{x}$ is not equal to $i_{x}$, changing $g_{x}$ by $d_{x}$ levels cannot be compensated with changing $g_{y}$ by $d_{y}$ levels. $F$ is not linear because people have different "mental" spending accounts for different goals [17], i.e., if a stakeholder reaches the maximum amount that is willing to spend on satisfying a goal $g_{x}$, he/she will not sacri ce other goals for $g_{x}$, if $g_{x}$ is already highly satis ed. Even though stakeholders may have previously increased the satisfaction level of $g_{x}$ and compensated it by decreasing other goals, but as soon as they psychologically feel that too much is spent on $g_{x}$, the MRS they are willing to spend on $g_{x}$ would change in the next Even Swaps queries.

For example, if stakeholders previously made a swap as $\left(T_{1}: 3 m s \rightarrow 2 m s \Leftrightarrow C_{1}: \$ 1.9 m \rightarrow \$ 2 m\right)$, it does not indicate that whenever $T_{1}$ is reduced $1 \mathrm{~ms}$, then, stakeholders are willing to pay an extra $\$ 0.1 \mathrm{~m}$. For instance, if humans cannot sense the difference between the $2 \mathrm{~ms}$ and $1 \mathrm{~ms}$ delays, reducing $T_{1}$ to less than $2 \mathrm{~ms}$ does not provide any extra value on performance. Therefore, the swap $\left(T_{1}: 2 m s \rightarrow 1 m s \Leftrightarrow C_{1}: \$ 1.9 m \rightarrow \$ 2 m\right)$ is not valid and reusable. This limits the reusability of swaps and increases the number of swap queries needed.

Method Feasibility. Making trade-offs by even swaps may need substantial cognitive abilities and domain knowledge. If stakeholders are not able to swap suggested goals, the tool suggests the next best swap, and stores the rejected swap in a "black list", and in the rest of the process, would not ask that swap from the stakeholder. In practice, stakeholders may reject every suggested swap, which results in several tedious swap queries to nd the one that stakeholder is able to answer. The process may become time-consuming or never ends.

To prevent the dead-ends, after a number of rejected suggestions, the tool asks the users to select the goals for the next swap themselves. Nevertheless, if stakeholders are not able the specify the maximal amount of a goal satisfaction level that they are willing to sacri ce for a unit of increase in another goal, then it is probable that they will not be able to numerically specify preferences over the goals either.

We have also assumed that stakeholders would make consistent swaps among goals. In practice, however, stakeholders are not always consistent in their judgements. As future work, we intend to enhance our tool to trace the inconsistencies in even swaps.

Another threat to practicality of the algorithm is the diversity of evaluation scales in the consequence table. Stakeholders may not be able to swap a goal measured in absolute values with a goal that is evaluated by qualitative labels such as $\checkmark$ and $x$. In future work, we intend to conduct empirical experiments to examine which types of swaps are easy/hard to make.

\section{RELATED WORK}

Multi-Criteria Decision Analysis (MCDA) methods have been successfully applied in the elds of economics, operation research, and management science, and can potentially be used in RE for deciding on alternative solutions to satisfy requirements. For example, requirements decision methods, such as [1], [7], [18], [8], [9], [10], rely on the availability, accuracy, and meaningfulness of quantitative costs and bene ts measures for making decisions.

Feather et al. [1] propose a quantitative model for strategic decision analysis and trade-off analysis considering quality requirements, by the "coarse quanti cation" of relevant risk factors and their interactions. Letier and Lamsweerde [10] argue that due to the lack of accuracy and measurability of goal formulations and lack of impact propagation rules through goal models, domain-speci c quality variables are needed to reason on partial goal satisfaction.

Utility values are used for representing quality value of NFRs in [19]. In the Utility Theory (Keeney and Raiffa [15]) the quantitative preference and measures are conjointly used to calculate the total utility value for each alternative. Preference (utility) elicitation involves a sequence of queries and interactions with the decisions stakeholders to obtain enough information about individual preferences. AHP [12] is a theory of relative measurement of intangible criteria to derive a numerical scale of priorities (preferences, importance weights) from pairwise comparisons of elements. Karlsson et al. [18] apply AHP for prioritizing software requirements and a cost-value analysis. A case study of applying AHP for software requirements prioritizing is given by Karlsson in [20].

The Even Swaps [13] method avoids eliciting explicit numerical priorities, and yet incorporates the stakeholders' preferences in determining the optimum solution by querying value trade-offs between stakeholders' goals.

The need for automated tool support for suggesting swaps to decision analysts has been recognized [21], [14]. In [21], consequence changes made in an even swap are not assumed to depend on the satisfaction levels of the goals, which 
allows using the trade-off information given in an even swap to represent the stakeholders' general preferences over the goals. The Smart Swaps tool [14] suggests next swaps to the user based on preference programming assumptions made in [21]. The Smart Swaps tool navigates the swap chains toward 1) making goals irrelevant and 2) making alternatives one step closer to the dominance situation.

In the current work, in addition to those two goals, we introduce other criteria for suggesting the next swaps, such as reusability of swaps, swaps that are cognitively easy to make, preferring numerical scales to qualitative ones, etc. Our tool is tailored for requirements trade-off analysis, e.g., the tool suggests swaps that stakeholders are willing to make among requirements with minimal sacri ce to be made on requirements. A demo of the proposed tool in this paper is available at [22].

The algorithm in the current work is developed based on the opposite assumption made in [21], i.e., we assume that consequence changes made in an even swap do not represent stakeholders' general preferences. In Section VI, we justi ed this assumption and explained its implication on the proposed algorithm.

\section{CONCLUSIONS}

In this work, we adopt and enhance the Even Swaps [13] method for analyzing trade-offs among requirements when multiple alternative design solutions satisfy different requirements to some extent. The main contribution of this paper is proposing a set of rules for suggesting next swaps to the decision stakeholders. The algorithm and prototype tool handle different types of input data: absolute and ordinal values in different scales.

\section{ACKNOWLEDGMENT}

Financial support from the Natural Sciences and Engineering Research Council of Canada is gratefully acknowledged. Authors thank Jocelyn Simmonds and Jennifer Horkoff for insightful comments on this work. We would like to thank ONE-ITS project members specially Roger Browne at Ministry of Transportation, Ontario (MTO), Professor Tamer El-Diraby and Mahmoud Osman Abou-Beih.

\section{REFERENCES}

[1] M. S. Feather, S. L. Cornford, K. A. Hicks, J. D. Kiper, and T. Menzies, "A broad, quantitative model for making early requirements decisions," IEEE Software, vol. 25, pp. 49-56, 2008.

[2] E. Yu, "Modeling Strategic Relationships for Process Reengineering," Ph.D. dissertation, University of Toronto, 1995.

[3] P. Giorgini, J. Mylopoulos, E. Nicchiarelli, and R. Sebastiani, "Formal reasoning techniques for goal models," Journal of Data Semantics, vol. 1, pp. 1-20, 2003.

[4] L. Chung, B. A. Nixon, E. Yu, and J. Mylopoulos, NonFunctional Requirements in Software Engineering. Kluwer Academic, 1999.

[5] J. Horkoff and E. Yu, "A Qualitative, Interactive Evaluation Procedure for Goal- and Agent-Oriented Models," in CAiSE Forum, 2009.
[6] P. Giorgini, J. Mylopoulos, and R. Sebastiani, "Goal-oriented requirements analysis and reasoning in the tropos methodology," Eng. Appl. Artif. Intell., vol. 18, no. 2, pp. 159-171, 2005.

[7] W. Ma, L. Liu, H. Xie, H. Zhang, and J. Yin, "Preference model driven services selection," in Proc. of CAiSE'09, 2009, pp. 216-230

[8] P. Giorgini, G. Manson, and H. Mouratidis, "On security requirements analysis for multi-agent systems." in SELMAS'03, 2003.

[9] Y. Asnar and P. Giorgini, "Modelling risk and identifying countermeasure in organizations," 2006, pp. 55-66.

[10] E. Letier and A. van Lamsweerde, "Reasoning about partial goal satisfaction for requirements and design engineering," in SIGSOFT '04/FSE-12, 2004, pp. 53-62.

[11] H. P. In, D. Olson, and T. Rodgers, "Multi-criteria preference analysis for systematic requirements negotiation," in Proc. of the COMPSAC'02, ser. COMPSAC '02, 2002, pp. 887-892.

[12] T. Saaty, The Analytic Hierarchy Process, Planning, Piority Setting, Resource Allocation. New york: McGraw-Hill, 1980.

[13] J. S. Hammond, R. L. Keeney, and H. Raiffa, Smart choices : a practical guide to making better life decisions. Broadway Books, 2002.

[14] J. Mustajoki and R. P. Hämäläinen, "Smart-swaps - a decision support system for multicriteria decision analysis with the even swaps method," Decis. Support Syst., vol. 44, no. 1, pp. 313-325, 2007.

[15] R. L. Keeney and H. Raiffa, Decisions with multiple objectives : preferences and value tradeoffs. Wiley, 1976.

[16] J. Yen and W. A. Tiao, "A systematic tradeoff analysis for con icting imprecise requirements," in $R E$ '97, 1997, p. 87.

[17] B. Schneier, "The psychology of security," Commun. ACM, vol. 50 , no. 5 , p. $128,2007$.

[18] J. Karlsson and K. Ryan, "A cost-value approach for prioritizing requirements," IEEE Softw., vol. 14, no. 5, pp. 67-74, 1997.

[19] H. Xie, L. Liu, and J. Yang, "i*-prefer: optimizing requirements elicitation process based on actor preferences," in $S A C$ '09: Proceedings of the 2009 ACM symposium on Applied Computing, 2009, pp. 347-354.

[20] J. Karlsson, "Software requirements prioritizing," In 2nd IEEE International Conference on Requirements Engineering, pp. 100-116, 1996.

[21] J. Mustajoki and R. P. Hämäläinen, "A preference programming approach to make the even swaps method even easier," Decision Analysis, vol. 2, no. 2, pp. 110-123, 2005.

[22] (2011) Decision analysis tool demo, release 1, dcs, univ. of toronto, available at http://www.cs.toronto.edu/gelahi/Release 1/Release1.html. 